
\title{
R Reserach S Suare \\ Establishment of a Rapid and Stable Transgenic System by Agrobacterium-Mediated Transformation of the Germination Seeds in Diploid Strawberry.
}

Xiao-Jiao Gu

Beijing University of Agriculture

Yuanyue Shen ( $\nabla$ sfmn@tom.com )

Beijing University of Agriculture https://orcid.org/0000-0001-5415-3174

\section{Methodology}

Keywords: Strawberry, Agrobacterium-mediated transformation of germinating seeds, reporter gene, CHLH, RNAi

Posted Date: July 2nd, 2020

DOI: https://doi.org/10.21203/rs.3.rs-38938/v1

License: (c) (1) This work is licensed under a Creative Commons Attribution 4.0 International License. Read Full License 


\section{Abstract}

\section{Background}

Strawberry (Fragaria) is regarded as a model plant for both Rosaceae and non-climacteric fruit ripening. Although much progress has been made in the identification of gene function using traditional, stable and transient genetic transformation systems in strawberry, the limitation is, more or less, present. Thus, development of a rapid, efficient, and stable transformation system is required for strawberry research and breeding.

Results

Here, using diploid Hawaii-4 (Fragaria vesca) seeds and a reporter gene of $\mathrm{CHLH}$ (the $\mathrm{H}$ subunit of magnesium chelatase magnesium chelatase), we first develop a new, rapid, efficient, and stable transgenic system by the Agrobacterium-mediated seed transformation to silence the reporter gene, obtaining a transformation frequency with $10 \%$ through a series of optimization conditions, including full imbibition and initial germination, shaking infection for $24 \mathrm{~h}$, dark cultivation on MS medium for $3 \mathrm{~d}$ at 24 ${ }^{\circ} \mathrm{C}$, light culture on MS-Tim medium for 1 week at $24{ }^{\circ} \mathrm{C}$, and vector construction carried fluorescence label. Taken together, radicle-emergence germination seeds, appropriate Agrobacterium concentration and infection time are critical for successful transformation, obtaining transgenic kanamycin-resistant seedlings within 1 month and T2 generation transgenic plants within 4 months.

Conclusions

We first have successfully established Agrobacterium-mediated transformation of germinating seeds (AMTGS) in diploid strawberry ( $F$. vesca), providing a useful tool for studying non-climacteric fruit ripening and strawberry breeding.

\section{Background}

Strawberry (Fragaria), as a distinct member of Rosaceae, is not only an important fruit crop worldwidely, but is also regarded as a model for studying non-climacteric fruit ripening [1]. In the past years, a large of genes related to strawberry fruit ripening have been identified via traditional stable and transient transgenic systems in strawberry; however, more or less, the limitation is existed [2-4]. Thus, development of a rapid, efficient, and stable transformation system is required for strawberry research and breeding.

It is known that strawberry leaf tissue has been extensively studied to develop efficient transformation and regeneration methods through somatic tissue or callus formation [5-14]. However, the regeneration of transgenic strawberry plants not only remains difficult, but also takes a long time from transformation to identification $[3,15,16]$. Such as, it takes at least 15 months from transformation to gaining transgenic fruits for gene function analysis in ripening $[17,18]$. In addition, although the transient transformation 
technology in strawberry fruits provides a rapid tool to study gene function in ripening, some limits are existent in fruit transient transgenic system $[2,4]$.

In addition to the widely-used leaf-disk transformation $[14,19,20]$, other tissues are also used for gene transformation21-30. The mature and immature embryo-generated calli are used to develop transgenic plants in rice, maize, wheat and Barley [21-23, 27]. Notably, a non-tissue culture-based method, floral dip transformation, is widely and efficiently used in Arabidopsis as a model system [24-26]. Notably, the Agrobacterium-mediated transformation of germinating seeds (AMTGS) of Arabidopsis, another nontissue culture approach is also early reported [31]. In recent years, the AMTGS of kenaf (Hibiscus cannabinus) seeds have been developed within 3 months with $6 \%$ transformation efficiency by the efficient, fast, reliable stable, non-tissue culture transgenic method [32]. Therefore, to rapidly identify gene function in strawberry, an AMTGS of diploid strawberry 'Hawaii-4' (Fragaria vesca) is to be established.

It is known that selection of a good reporter gene contributes to early identification of transgenic lines. In the past years, the $\mathrm{H}$ subunit of magnesium chelatase magnesium chelatase (CHLH), an enzyme involved in chlorophyll biosynthesis, is chosen as a good reporter gene, in that its silencing phenotype was clearly observed through chimeric phenotypes with yellow/white leaves [33-36]. In the present study, on the basis of CHLH function in chlorophyll biosynthesis, an Agrobacterium-mediated transformation of germinating seeds in diploid strawberry was first established.

\section{Results}

\section{Determination of seed imbibition extent and optimum kanamycin screening concentration}

To establish rapid and stable transgenic system in strawberry, the Agrobacterium-mediated transformation of germinating seeds in diploid strawberry was first studied based on the previous reports $[31,32]$. We first find that there was no significant difference in the germination rate of strawberry seeds under different light culture conditions, including light culture, dark culture, and light culture after dark culture; the three treatments had a few, half, and $90 \%$ of seed germination respectively with $4-d, 5-d$ and 6-d imbibition (Fig. 1a).

To confirm optimal kanamycin concentration for screening, the sterilized strawberry seeds were cultured in MS selection medium with kanamycin concentration at $0,25,50,75,100 \mathrm{mg} / \mathrm{L}$ for 15 days. The results showed that when the concentration of kanamycin was $0 \mathrm{mg} / \mathrm{L}, 95 \%$ of the seeds germinated, the plants grew normally and the leaf color was dark green. Compared with no selection pressure, when the concentration of kanamycin increased to $25 \mathrm{mg} / \mathrm{L}, 91.7 \%$ of the seeds germinated, except for the growth of the root system is slightly slow, the germinated seedlings continued to show as the same as the wildtype growth; when the concentration of kanamycin was $50 \mathrm{mg} / \mathrm{L}, 41.7 \%$ of the seeds germinated, but the first leaf of the germinated seedlings could not fully unfold; when the selection pressure was increased to $75 \mathrm{mg} / \mathrm{L}$, only $20 \%$ of the seeds could germinate and the color of the two cotyledons of the seedlings 
were yellow and white; when the selection pressure increased to $100 \mathrm{mg} / \mathrm{L}, 5 \%$ of the plants germinated and died of browning (Fig. 1b, C). Taken together, $75 \mathrm{mg} / \mathrm{L}$ of kanamycin in MS-selection medium was used as an antibiotic selection.

\section{Optimization of infection parameters}

After 3-d culture in the selection medium, the GFP could be observed in the successfully-transgenic plants (Fig. 2a, b). Similarly, the unsuccessfully-infected seedlings had no GUS expression (Fig. 2c, e), while the successfully-infected seedlings showed blue in the roots and true leaves with GUS expression (Fig. 2d, f). Base on the observation of both GFP and GUS expression in transgenic seedlings, a 1.5-OD600 value of the Agrobacterium infection solution was optimum infection concentration (Fig. $2 \mathrm{~g}$ ). The seeds with just sprouted the radicles had highest infection efficiency (Fig. 2h) during 24-h infection (Fig. 2i). Taken together, the optimum parameters of Agrobacterium-mediated transformation of germinating seed in diploid strawberry are as follows: $75 \mathrm{mg} / \mathrm{L}$ of kanamycin in MS-selection medium, the seeds with just sprouted the radicles, 24-h infection in 1.5-OD600 value of the Agrobacterium infection solution, and then it was dark cultured at $24{ }^{\circ} \mathrm{C}$ for $3 \mathrm{~d}$.

Establishment of a rapid and stable transgenic system by the Agrobacterium-mediated transformation of germinating seeds by a reporter gene of FVCHLH in strawberry

The $\mathrm{CHLH}$ encoding the $\mathrm{H}$ subunit of magnesium chelatase magnesium chelatase involved in chlorophyll biosynthesis is chosen as a reporter gene to verify the effectiveness of the seed transformation system owing to its silencing phenotypes with yellow/white leaves easy to be observed [33-36].

Based on the optimal transformation conditions obtained above, we have transformed the germination seeds with just sprouted the radicles of diploid strawberry 'Hawaii-4' through the GV3101 strain carrying the pK7GWIWG2 (II) RR - FvCHLH vector. After 3-day infection under dark culture, the seeds were transferred to MS-selection medium. After 3 days, the seeds were screened by DsRed fluorescence. After 2 weeks, the fluorescent seedlings were transferred to quartz sand and watered using nutrient solution containing $75 \%$ kanamycin. The whole processes for transformation of germination strawberry seeds were shown in Fig. 3. We found that after observation with fluorescence microscope, the fluorescence of DsRed + plants showed an enhanced trend. The fluorescence of 7-day-old plants (Fig. 4c, d) cultured on MS-selection medium was significantly higher than that of 3-day-old plants (Fig. 4a, b). DsRed fluorescence was also expressed in the roots (Fig. 4e, f) and stolons (Fig. 4i, j) of the plants after three months, but not in the wild-type plants of the roots (Fig. 4g, h) and stolons (Fig. 4k, l).

One month after transplanting, in comparison to the control, the FVCHLHRNAi plants showed yellow spots (Fig. 5a, phenotype-1), or half green and half white in a leaf (Fig. 5a, phenotype-2). The PCR was performed to detect both kanamycin and DsRed gene fragments in the T-DNA inserted, transgenic plants. The expected 400-bp Kana and 964-bp DsRed products were detected in five FvCHLH-RNAi plants but not in the wild-type plants (Fig. 5b, c). Compared with the wild-type plants, the expression of FvCHLH gene in the FVCHLHRNAi plants was significantly down regulated (Fig. 5d). In the process of transformation, 
phenotype- 1 plants accounted for $10 \%$ of the total infected plants, and phenotype- 2 plants accounted for about $20 \%$ of the total infected plants, it can be concluded that the conversion efficiency is over $10 \%$ (Fig. 5e). These results showed that through Agrobacterium-mediated transformation of germinating seed, the pK7GWIWG2 (II) RR-FVCHLH vector was successfully transformed and inhibited FVCHLH expression, and the FVCHLHRNAi transgenic plants have chlorosis phenotypes.

\section{T2 seedlings of FvCHLH-RNAi plants retains kanamycin- resistance}

T1 seeds of $F v C H L H$ RNAi transgenic plants were spread on the quartz sand soaked in the nutrient solution containing $75 \mathrm{mg} / \mathrm{L}$ kanamycin. In the presence of kanamycin, the seeds of sensitive to kanamycin cannot germinate or the cotyledons turn yellow 10 days after germination, and the true leaves could not develop. T2-resistant seedlings could germinate on quartz sand with kanamycin and continued to grow normally (Fig. 6a). The Kanamycin resistant bands and DsRed bands were detected in T2 generations (Fig. 6b). These results demonstrate that the transgenic plants can obtain by seed transformation and the relative genetic characteristics may be transferred to T1 progeny. A rapid and stable transgenic system by the Agrobacterium-mediated transformation of germinating seed is successfully established.

\section{Discussion}

Strawberry is a model plant for both Rosaceae and non-climacteric fruit ripening. Although much progress has been made underlying the molecular mechanism of strawberry fruit ripening using transiently-genetic fruit transformation and stably-genetic transformation systems, in which some limits are existed [1-14, 16, 37]. Notably, it is previously reported that the Agrobacterium-mediated seed transformation may facilitate the entry of Agrobacterium tumefaciens into the seeds to integrating a target gene into the embryo cells [31-32], similar to the pollen-tube mediated genetic transformation method widely used in Arabidopsis thaliana [24, 30]. Reference of the seed transformation method [3132], we first establish the Agrobacterium-mediated transformation of the germination seeds in diploid strawberry by a series of optimization conditions.

First, some factors key to transformation efficiency were confirmed, including seed imbibition time, suspension Agrobacterium concentrations, Agrobacterium infection time, kanamycin concentration in selection of transgenic plants. (1) Full imbibition is requisite for seed transformation. It is early reported that in Arabidopsis seed transformation, 12-h imbibition has the highest transformation efficiency, less than $9 \mathrm{~h}$ showing no transformants [31]. In strawberry, we demonstrate that seed imbibition to reach both testa rupture and, radicle emerging is vital for transformation efficiency (Fig. 2). In addition, Agrobacterium concentrations and infection time are also critical to transformation efficiency [38]. We also confirm that 24-h infection time has maximum transformation efficiency, in consistent with the previous reports in seed transformation [31, 32]. Generally, in the conventional leaf disk method, Agrobacterium with concentration at $0.3-0.5$ of OD 600 has high vigor and is used as an optimal 
concentration for infection [39], in that a higher Agrobacterium concentration causes harm to explant differentiation including browning even death, and a lower concentration is not enough Agrobacterium for T-DNA integration of explants with lower transformation efficiency. In the present study, we find that the germination seeds were more tolerant to Agrobacterium tumefaciens, and OD600 of 1.5 is an optimal concentration for highest transformation efficiency (Fig. 2). Finally, the appropriate concentration of kanamycin is also pivotal for selection of transgenic positive plants. In Arabidopsis seed transformation, $\mathrm{T} 1$ and T2 generation positive seedlings could be selected on medium containing $100 \mathrm{mg} / \mathrm{L}$ kanamycin [31]. In Kenaf seed transformation, F0 and F1 plants were successfully obtained on medium containing $50 \mathrm{mg} / \mathrm{L}$ kanamycin [32]. In the present study, we find that strawberry seedlings were selected on medium containing $75 \mathrm{mg} / \mathrm{L}$ kanamycin, in which positive transgenic plants could developed true leaves, whereas not in the control (Fig. 2). Taken together, we confirm high efficiency transformation parameters in the Agrobacterium-mediated transformation of the germination seeds of diploid strawberry, including radicle initially-emerging seeds, $1.5-\mathrm{OD}_{600}$ Agrobacterium, 24 -h infection, and $75 \mathrm{mg} / \mathrm{L}$ kanamycin in transgenic plant selection.

In fact, how to early select positive transgenic seedlings is more important in genetic transformation. It is previously demonstrated that the reporter gene is a good strategy for early transformation selection, such as neomycin phosphotransferase gene (NPT区), green fluorescent protein (GFP), red fluorescent protein (DsRed), and $\beta$-glucuronidase (GUS) genes [31, 40]. In the present study, apart from the GFP and GUS genes (Fig. 2), we also used the reporter gene encoding magnesium chelatase $\mathrm{H}$ subunit (CHLH) functioned in chlorophyll biosynthesis, in that when its expression is downregulated, resulting in yellow or white leaf phenotype and thus using as a good report gene [33-36]. We construct the recombination pK7GWIWG2 (II) RR-FvCHLH vector with two reporter genes, DsRed and CHLH, which used as early selection. On the basis of DsRed fluorescence, it is easy to pick out early potential transgenic seedlings, which is further confirmed to be positive transgenic plants by kanamycin selection (Figs. 3 and 4). The first selection by DsRed fluorescence may bypass a large quantity of seeds used for kanamycin selection, not only saving more time and labor, but also and making it easier to obtain transgenic plants. One month after the transformation, through the reporter gene $\mathrm{CHLH}$, we may observe the leaves of transgenic plants with a loss-of-green phenotype (Fig. 5). In all, through the two reporter genes, the Agrobacteriummediated transformation of strawberry seeds was successfully established.

To further confirm the Agrobacterium-mediated transformation of the germination seeds, we also carried out kanamycin resistance and PCR analysis in T2 seedlings (Fig. 6): (a) when strawberry seedlings were selected on medium containing $75 \mathrm{mg} / \mathrm{L}$ kanamycin, the true leaves of kanamycin-resistant $\mathrm{T} 2$ seedlings can develop normally, whereas the growth of wild-type seedlings and kanamycin-sensitive T2 seedlings was partially inhibited and true leaves failed to develop; (b) through PCR test, the integration of DsRed gene and Kanamycin resistance gene into the genome of kanamycin-resistant T2 seedlings have been confirmed, whereas in wild-type seedlings and kanamycin-sensitive T2 seedlings, corresponding gene integration has not been detected. By analysis of the transgenic characteristics of transformed progeny 
(T1 and T2 generation), we provide strong evidence to confirm that the Agrobacterium-mediated transformation of strawberry seeds was successfully established.

In summary, we have first established a fast and efficient protocol for Agrobacterium-mediated transformation of strawberry seeds: (1) seed imbibition time (just to the point of radicle emergence) is a key step; (2) Agrobacterium concentration and the infection time are also critical for successful transformation; (3) the selection of kanamycin-resistant seedling takes 1 month, T2 generation transgenic plants are obtained within 4 months; (4) the transformation efficiency is $10 \%$. Thus, this method can greatly shorten the experimental cycle and simplify the operation processes. Give that strawberry is a model plant for both Rosaceae and non-climacteric fruit ripening, the Agrobacteriummediated transformation of strawberry seeds is to be widely used and will facilitate fruit development research and breeding.

\section{Conclusions}

On the basis of the CHLH (the H subunit of magnesium chelatase magnesium chelatase) as a reporter gene and the germinating seeds with radicle appearance, the Agrobacterium-mediated transformation of germinating seeds (AMTGS) in diploid strawberry (Fragaria vesca) has first been established, especially finding that appropriate Agrobacterium concentration and infection time are critical for the transformation. The successful transgenic kanamycin-resistant seedlings (T1) are gained within 1 month and T2 transgenic plants within 4 months. In all, this new method is to provide a powerful tool for rapid identification of gene function in strawberry.

\section{Methods}

\section{Plant materials and seeds germination}

The diploid strawberry 'Hawaii-4' (Fragaria vesca) plants were grown in green house at $25 \pm 1{ }^{\circ} \mathrm{C}$. The seeds were taken from ripe fruits and residual receptacle was removed, then the seeds were put at room temperature to dry for 1-2 d. The dried seeds are put into 2-mL tube and stored at $4{ }^{\circ} \mathrm{C}$ for use. Seeds were sterilized by $1 \%$ sodium hypochlorite for $8 \mathrm{~min}$ and washed with sterile water three times. The sterilized seeds were transferred onto MS-germination medium [4.43 g/L MS (Phyto Technology Laboratories M519), 2\% sucrose (Sangon, China), $0.8 \%$ agar (Sangon, China), pH 5.8], and left at $4{ }^{\circ} \mathrm{C}$ for $24 \mathrm{~h}$ to break dormancy. The dormancy-broken seeds were treated with three test treatments: light culture in a light incubator (PRX-350C, Sdfu, China), dark culture wrapped with tin foil, and light culture after 2 days of dark culture. The seed culture condition in light incubator is as a $16 \mathrm{~h}$ photoperiod with a light intensity of $30,000 \mathrm{LX}(250 \mu \mathrm{mol} \cdot \mathrm{m}-2 \cdot \mathrm{s}-1)$ at $24{ }^{\circ} \mathrm{C}$. Each treatment was inoculated with 20 seeds (three repeats) for germination.

\section{Vector construction and Agrobacterium tumefaciens culture}


Two Agrobacterium strains were used to optimize transformation parameters, namely GV3101 strain carrying pCAMBIA 1300 vector with a Green fluorescent protein (GFP) reporter gene and carrying pBI121 vector with a $\beta$-glucuronidase (GUS) reporter gene. The RNAi recombinant plasmid was constructed using the Gateway system and target genes were silenced in strawberry fruit by intron splicing RNA (ihpRNA). The 465-bp FvCHLH were amplified and cloned into the pDONRTM 221 vector (Invitrogen, Beijing China) then into the pK7GWIWG2 (II) RR vector using Gateway LR Clonase II enzyme (Invitrogen 11791-020, USA) combination technology. The primers for constructing FVCHLH-RNAi were: forward, 5'GGGGACAAGTTTGTACAAAAAAGCAGGCTTCGCTGCCAACAATCCATCT-3' and reverse, 5'GGGGACCACTTTGTACAAGAAAGCTGGGTCTCTTCCAACTGTCTCAGCCA-3'. The pK7GWIWG2 (II) RR vector contains the red fluorescence protein (DsRed) reporter gene and kanamycin resistance gene.

The recombinant plasmid was transformed into A. tumefaciens GV3101, and a single colony was picked

and placed in Luria broth (LB) medium containing antibiotic $20 \mu \mathrm{g} / \mathrm{mL}$ rifampicin (Sangon, A600812, China), $50 \mu \mathrm{g} / \mathrm{mL}$ gentamicin (Sangon, A100304, China), and $100 \mu \mathrm{g} / \mathrm{mL}$ spectinomycin (Sangon, B541016, China) at $28{ }^{\circ} \mathrm{C}$, and $180 \mathrm{rpm}$ overnight. The single colony of GV3101 strains (GV3101 strain of pCAMBIA 1300 and GV3101 strain of pBI121) were put into an LB medium containing antibiotics $20 \mu \mathrm{g} / \mathrm{mL}$ rifampicin, $50 \mu \mathrm{g} / \mathrm{mL}$ gentamicin, $100 \mu \mathrm{g} / \mathrm{mL}$ kanamycin (Sangon, A506636, China) at $28{ }^{\circ} \mathrm{C}$, and $180 \mathrm{rpm}$ overnight. Above three kinds of bacteria liquid were collected by centrifugation at 6,000 rpm for $5 \mathrm{~min}$, and suspended using MS-infection [4.43 g/L MS, 2\% sucrose, $200 \mu \mathrm{mol} / \mathrm{L}$ Acetosyringone (As, Sigma-Aldrich, D134406, USA)], pH 5.8 to prepare an Agrobacterium infection solution.

\section{Determination of appropriate Kanamycin concentration for screening}

The sterilized seeds were cultured on the MS-selection medium containing different concentrations of kanamycin with $0,25,50,75$ and $100 \mathrm{mg} / \mathrm{L}$ in order to determine most suitable antibiotic screening concentration. Twenty seeds were placed in each petri dish and the experiment were done with three repetitions.

\section{Optimization of parameters related to infection efficiency}

The pre-imbibed seeds were placed in Agrobacterium infection solution and co-cultured at $120 \mathrm{rpm}, 28^{\circ} \mathrm{C}$ for $24 \mathrm{~h}$, then the seeds were placed in MS-germination medium and wrapped in tin foil at $25^{\circ} \mathrm{C}$ for $3 \mathrm{~d}$. Subsequently, the seeds were further placed in MS-selection medium $[4.43 \mathrm{~g} / \mathrm{L} \mathrm{MS}, 2 \%$ sucrose, $75 \mathrm{mg} / \mathrm{L}$ kanamycin, $200 \mathrm{mg} / \mathrm{L}$ Timentin (Phyto Technology Laboratories T869), 0.8\% agar, pH 5.8]. After 3 days, GFP/DsRed fluorescent tag and GUS reporter gene were used for early screening and statistics. After 2 weeks, the seedlings with fluorescence were transplanted into quartz sand and covered with cling film for three days to prevent water loss, and watered with the kanamycin-added nutrient solution to allow the seedlings to grow under appropriate selection pressure at condition with a greenhouse at $24{ }^{\circ} \mathrm{C}$, light/dark 16/8 h. 
To determine factors related to infection efficiency, we performed a series of experiments, including OD values (optical density at $600 \mathrm{~nm}, \mathrm{OD} 600=0.5,1,1.5$, and 2), infection time (12 h, $24 \mathrm{~h}$, and $36 \mathrm{~h}$ ), and seed germination status ( 1 represents the seed that has not yet exposed the radicle; 2 represents the seed that has just sprouted the radicle; 3 represents that the seed has two cotyledons,). The optimal infection parameters were determined by investigation of GFP using stereo fluorescence microscope (ZEISSAxiocam 506 color) and GUS Histochemical Stain Kit (Solarbio, G3060). Infection efficiency was calculated by the proportion of GFP fluorescent protein expression plants to the total infected plants or Gus expression plants to the total infected plants. During the infection process, 20 seeds were placed in each petri dish, and three replicates were performed.

\section{Identification of transgenic plants by PCR}

In order to confirm transformation of T-DNA in diploid strawberry genome, leaves of one-month-old transgenic strawberry plants with red light were collected and genomic DNA was extracted by Plant genomic DNA Extraction Kit (Aidlab Biotech, DN15, China). The leaves of wild type plants were used as control. The fragments of Kanamycin resistance genes and DsRed genes were amplified by PCR. Primers for PCR identification were: Kan forward, 5'-cgatagaaggcgat gcgctg-3' and reverse, 5'-

cgcttgatccggctacctg-3'; Red forward, 5'-cgcccttggtcaccttcagcttcac-3' and reverse, 5'caatgcagtgggacccacggttc-3'.

\section{Real-time PCR}

Total RNA from leaves of transgenic and wild-type plants was extracted using an OMEGA RNA extraction kit (OMEGA biotek, USA) according to manufacturer's protocols. To generate first-strand cDNA, $400 \mathrm{ng}$ of total RNA was reverse transcribed using the Trans kit (Transgen, China) according to manufacturer's protocols. The first-strand CDNA was used as a template for PCR amplification for real-time PCR on a Light Cycler 96 Real Time PCR System (Roche Diagnostics GmbH, Mannheim City, Germany) using TransStart Top Green qPCR SuperMix (Transgen, China). The reactions of $10 \mu \mathrm{L}$ contained $5 \mu \mathrm{L}$ qPCR SuperMix, $0.25 \mu \mathrm{L}$ forward specific primer (10 $\mu \mathrm{M}$; Sangon, China), $0.25 \mu \mathrm{L}$ reverse specific primer $(10 \mu \mathrm{M}$; Sangon, China), $2 \mu \mathrm{L}$ cDNA template and $2.5 \mu \mathrm{L} \mathrm{ddH2O}$. Actin was used as a reference gene. Relative gene expression was analyzed by LightCycler ${ }^{\circledR} 96$ SW 1.1. The primers used for real-time PCR were: forward, 5'-TGGGTCCCCTGATAAC-3'and reverse, 5'-CCAAATCCCACTGTCC-3'.

\section{Kanamycin resistance test of $\mathrm{T} 1$ seeds}

Twenty seeds were obtained from a single inbred T1 generation, sprinkled on quartz sand, and irrigated with kanamycin $(75 \mathrm{mg} / \mathrm{L})$. After 10 days of culture, the sensitivity of the plants to kanamycin resistance was observed, and DNA molecules were analyzed for the resistant seedlings.

\section{Declarations}

\section{Acknowledgments}

We thank PhD Robert McKenzie for editing the English text of a draft of this manuscript. 


\section{Funding}

This study was supported by the China National Science Foundation (Projects 31672125), the Beijing Natural Science Foundation (6171001), the Teams and Teacher Career Development for Universities and Colleges in the Beijing Municipality (Grant no. IDHT20140509).

\section{Availability of data and materials}

The datasets supporting the conclusions and a description of the complete protocols are included within the article.

\section{Ethics approval and consent to participate}

Not applicable.

\section{Consent for publication}

Not applicable.

\section{Competing interests}

The authors declare that they have no competing interests.

\section{Author information}

\section{Affiliations}

College of Plant Science and Technology, Beijing University of Agriculture, Beijing 102206 China.

Xiao-Jiao Gu, Yuan-Yue Shen

\section{Authors' contributions}

XJ performed the experiments. YY designed the research and wrote the article.

\section{Corresponding author}

Correspondence to Yuan-Yue Shen

e-mail: sfmn@tom.com

Tel/Fax: +861080794426

\section{References}


1. Li CL, Jia HF, Chai YM. Shen YY. Abscisic acid perception and signaling transduction in strawberry: A model for non-climacteric fruit ripening. Plant Signaling Behavior. 2011;6:1950-3.

2. Guidarelli $M$, Baraldi $E$. Transient transformation meets gene function discovery: the strawberry fruit case. Front Plant Sci. 2015;12(6):444.

3. Haddadi F, Aziz MA, Abdullah SN, Tan SG, Kamaladini H. An efficient Agrobacterium-mediated transformation of strawberry cv. Camarosa by a dual plasmid system. Molecules. 2015;20:3647-66.

4. Zhao Y, Mao W, Chen Y, Wang W, Dai Z, Dou Z, Zhang K, Wei L, Li T, Zeng B, Liu T, Fan Y, Yan J, Li B, Jia W. Optimization and standardization of transient expression assays for gene functional analyses in strawberry fruits. Horticulture Research. 2019;6:53.

5. Nehra NS, Chibbar RN, Kartha KK, Datla RSS, Crosby WL, Stushnoff C. Genetic transformation of strawberry by Agrobacterium tumefaciens using a leaf disk regeneration system. Plant Cell Rep. 1990;9:293-8.

6. El Mansouri L, Mercadi JA, Valpuesta V, Lopez-Aranda JM, Pliegi-Alfaro F, Quesada MA. Shoot regeneration and Agrobacterium -mediated transformation of Fragaria vesca L. Plant Cell Rep. 1996;15:642-6.

7. Haymes KM, Davis TM. Agrobacterium -mediated transformation of 'Alpine' Fragaria vesca and transmission of transgenes to R1 progeny. Plant Cell Rep. 1997;17:279-83.

8. Balokhina NV, Kaliayeva MA, BuryanovYa I. The elaboration of a shoot regeneration system for the genetic transformation of the wild strawberry (Fragaria vesca L.). Biotekhnologiya. 2000;16:46-51.

9. Jimenez-Bermudez S, Redondo NJ, Munoz-Blanco J, Caballero JL, Lopez-Aranda JM, Valpuesta V, Pliego-Alfaro F, Quesada MA, Mercado JA. Manipulation of strawberry fruit softening by antisense expression of a pectate lyase gene. Plant Physiol. 2002;128:751-9.

10. Agius F, Gonzalez-Lamothe R, Caballero JL, Munoz-Blanco J, Botella MA, Valpuesta V. Engineering increased vitamin $\mathrm{C}$ levels in plants by overexpression of a D-galacturonic acid reductase. Nat Biotechnol. 2003;21:177-81.

11. Oosumi T, Gruszewski HA, Blischak LA, Baxter AJ, Wadl PA, Shuman JL, Veilleux RE, Shulaev V. Highefficiency transformation of the diploid strawberry (Fragaria vesca) for functional genomics. Planta. 2006;223:1219-30.

12. Qin Y, Jaime A, da Silva T, Zhang L, Zhang S. Transgenic strawberry: state of art for improved traits. Biotechnol Adv. 2008;26:219-32.

13. Zhang Q, Folta KM, Davis TM. Somatic embryogenesis, tetraploidy, and variant leaf morphology in trasgenic diploid strawberry (Fragaria vesca subspecies vesca 'Hawaii 4'. BMC Plant Biol. 2014;14:23.

14. Song GQ, Prieto H, Orbovic V. Agrobacterium-Mediated Transformation of Tree Fruit Crops: Methods, Progress, and Challenges. Front Plant Sci. 2019;10:226.

15. Barcelo M, El-Mansouri I, Mercado JA, Quesada MA, Alfaro P. F. Regeneration and transformation via Agrobacterium tumefaciens of the strawberry cultivar Chandler. Plant Cell Tissue Organ Culture. 1998;54:29-36. 
16. Folta KM, Dhingra A. Invited review: Transformation of strawberry: The basis for translational genomics in Rosaceae. In Vitro Cellular Developmental Biology Plant. 2006;42:482-90.

17. Hoffmann T, Kalinowski G, Schwab W. RNAi-induced silencing of gene expression in strawberry fruit (Fragaria $x$ ananassa) by agroinfiltration: a rapid assay for gene function analysis. Plant $\mathrm{J}$. 2006;48:818-26.

18. Miyawaki K, Fukuoka S, Kadomura Y, Hamaoka H, Mito T, Ohuchi H, Schwab W, Noji S. Establishment of a novel system to elucidate the mechanisms underlying light-induced ripening of strawberry fruit with an Agrobacterium-mediated RNAi technique. Plant Biotechnology. 2012;29:271-7.

19. Horsch RB, Fry JE, Hoffmann NL, Eichholtz D, Rogers SG, Fraley RT. A simple and general-method for transferring genes into plants. Science. 1985;227:1229-31.

20. Cappelletti R, Sabbadini S, Mezzetti B. Strawberry (Fragaria x ananassa). Methods in Molecular Biology. 2015;1224:217-27.

21. Potrykus I. Gene transfer to cereals: an assessment. Trends Biotechnol. 1990;8:535-42.

22. Ishida Y, Saito H, Ohta S, Hiei Y, Komari T, Kumashiro T. High efficiency transformation of maize (Zea mays L.) mediated by Agrobacterium tumefaciens. Nat Biotechnol. 1996;14:745-50.

23. Ishida $Y$, Tsunashima M, Hiei Y, Komari T. Wheat (Triticum aestivum $L$.) transformation using immature embryos. Methods Mol Biol. 2014;1223:189-98.

24. Clough SJ, Bent AF. Floral dip: a simplified method for Agrobacterium-mediated transformation of Arabidopsis thaliana. The Plant Journal. 1998;16:735-43.

25. Bent A. Arabidopsis thaliana floral dip transformation method. Methods Mol Biol. 2006;343:87-103.

26. Zhang X, Henriques R, Lin SS, Niu QW. Chua N. H. Agrobacterium-mediated transformation of Arabidopsis thaliana using the floral dip method. Nature Protocol. 2006;1:641-6.

27. Hiei Y, Ohta S, Komari T, Kumashiro T. Efficient transformation of rice (Oryza sativa L.) mediated by Agrobacterium and sequence analysis of the boundaries of the T-DNA. Plant Journal for Cell Molecular Biology. 1994;6:271-82.

28. Harwood W. A. A protocol for high-throughput Agrobacterium-mediated barley transformation. Methods Mol Biol. 2014;1099:251-60.

29. Ratanasut K, Rod-In W, Sujipuli K. In planta Agrobacterium-mediated transformation of rice. Rice Sci. 2017;24:181-6.

30. Van Eck J. The Status of Setaria viridis Transformation: Agrobacterium-Mediated to Floral Dip. Front Plant Sci. 2018;9:652.

31. Feldmann KA, Marks MD. Agrobacterium-mediated transformation of germinating seeds of Arabidopsis thaliana: a non-tissue culture approach. Mol Gen Genet. 1987;208:1-9.

32. Hanana M, Ayad R, Mzid R, Khouja ML, Hanachi AS, Hamrouni L. Hanana M, Ayadi R. Mzid, Rim. Efficient method of seed transformation via Agrobacterium tumefaciens for obtaining transgenic plants of Hibiscus cannabinus L. Ind Crops Prod. 2018;113:274-82. 
33. Hiriart JB, Aro EM, Lehto K. Dynamics of the VIGS-mediated chimeric silencing of the Nicotiana benthamiana $\mathrm{ChlH}$ gene and of the tobacco mosaic virus vector. Molecular Plant Microbe Interactions. 2003;16:99-106.

34. Hiriart JB, Lehto K, Tyystjärvi E, Junttila T, Aro EM. Suppression of a key gene involved in chlorophyll biosynthesis by means of virus-inducing gene silencing. Plant Mol Biol. 2002;50:213-24.

35. Park SH, Choi H, Kim S, Cho WK, Kim KH. Development of Virus-Induced Gene Expression and Silencing Vector Derived from Grapevine Algerian Latent Virus. Plant Pathology Journal. 2016;32:371-6.

36. Pignatta D, Kumar P, Turina M, Dandekar A, Falk BW. Quantitative analysis of efficient endogenous gene silencing in Nicotiana benthamiana plants using tomato bushy stunt virus vectors that retain the capsid protein gene. Molecular Plant Microbe Interactions. 2007;20:609-18.

37. Folta KM, Davis TM. Strawberry genes and genomics. Crit Rev Plant Sci. 2006;25:399-415.

38. Marutani-Hert M, Bowman KD, McCollum GT, Mirkov E, Evens TJ, Niedz RP. A dark incubation period is important for Agrobacterium-mediated transformation of mature internode explants of sweet orange, grapefruit, citron, and a citrange rootstock. PloS One. 2012;7:10.

39. Zhao S, Ruan Y, Sun H, Wang B. Highly efficient Agrobacterium-based transformation system for callus cells of the C3 halophyte Suaeda salsa. Acta Physiol Plant. 2008;30:729-36.

40. Naylor LH. Reporter gene technology: The future looks bright. Biochem Pharmacol. 1999;58:749-57.

\section{Figures}


a
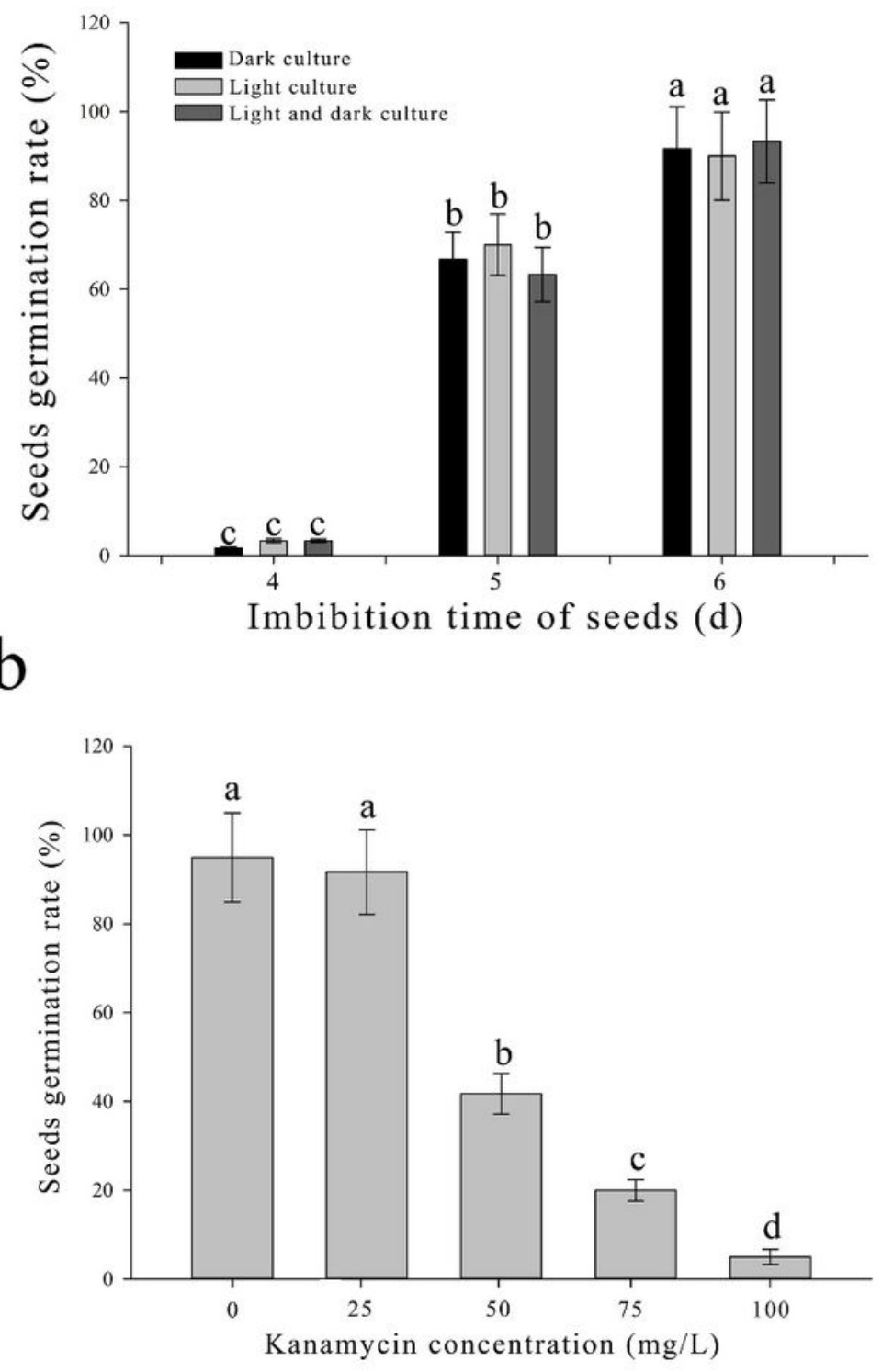

$\mathrm{C}$
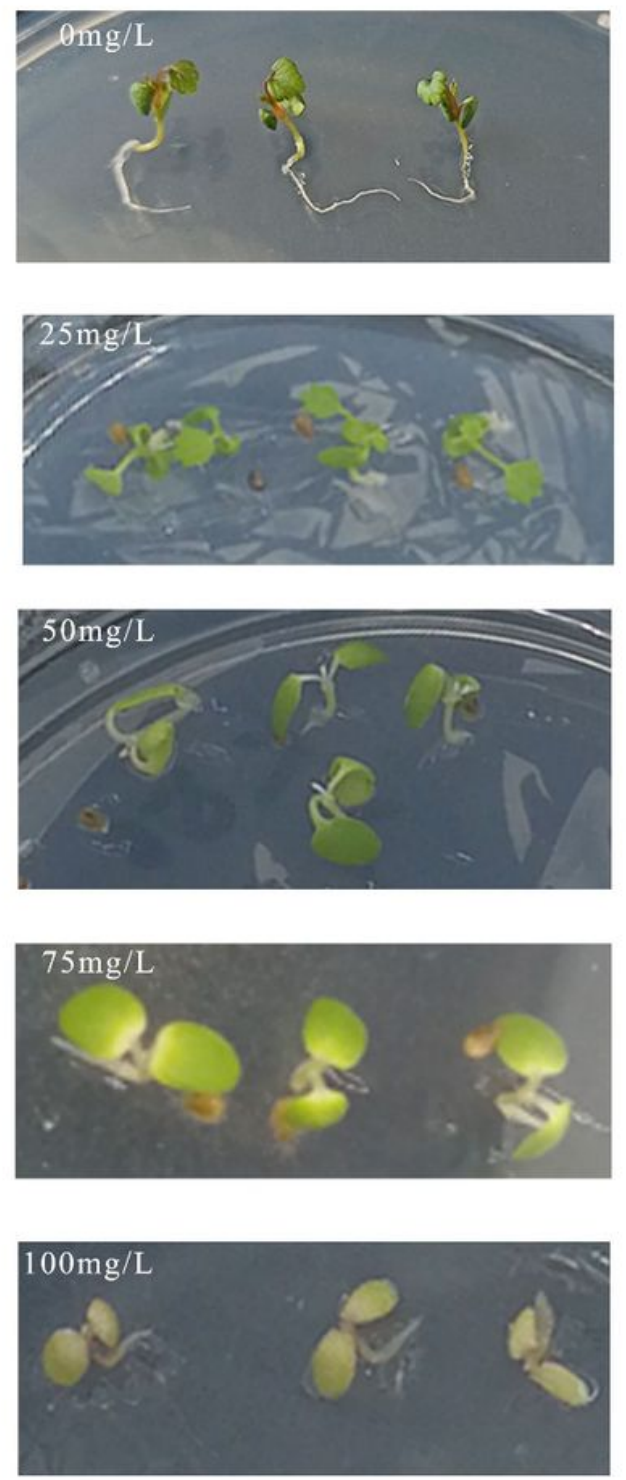

\section{Figure 1}

Determination of culture parameters in germination seeds and the optimal kanamycin screening concentration. a Seed germination rates in different light culture condition and imbibing time. b Seed germination rates in different kanamycin concentrations after 15-d cultivation. c Phenotypes in different kanamycin concentrations after $15-\mathrm{d}$ cultivation. Each treatment was treated with 20 seeds. The error bars represent the standard error $(n=3)$. Different letters indicate statistically significant differences at $\mathrm{P}<$ 0.05 as determined by Duncan's test. 

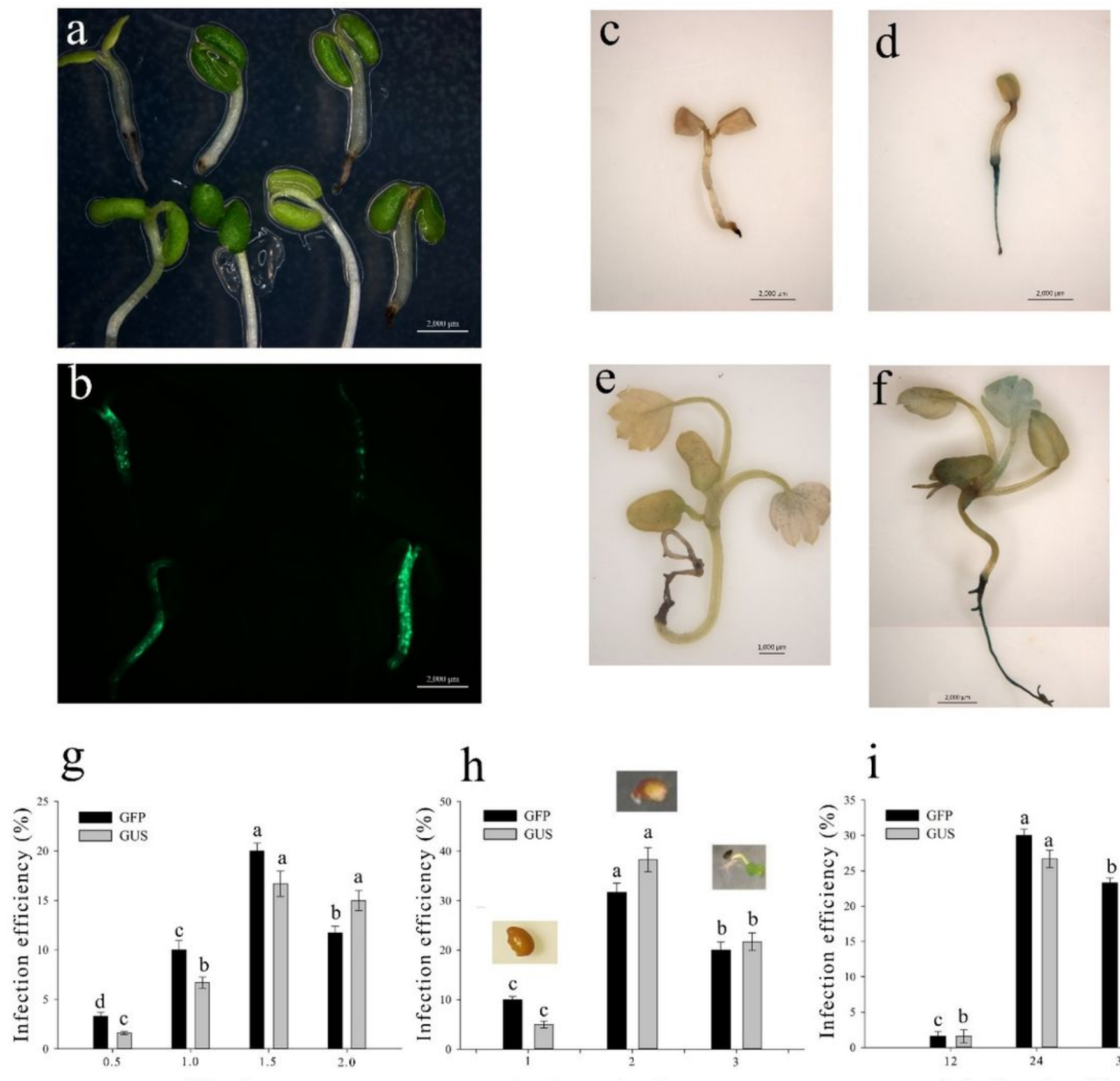

OD values

Seed germination states

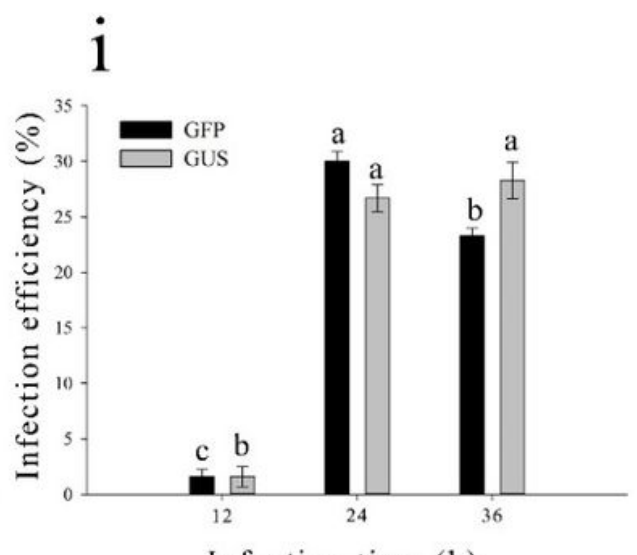

Infection time (h)

\section{Figure 2}

Determination of influence factors in Agrobacterium-mediated transformation of germinating seeds. a Infection seedlings after 3-d culture in MS-selection medium. b GFP expression in transgenic plants of Fig.2A. $c$ and e No GUS blue in the un-infection seedlings. $d$ and $f$ GUS blue in successfully-infected seedlings. $c$ and $d$ Infection seedlings after 3-d culture in MS-selection medium. e and f Infection seedlings after 2 weeks culture. g Effect of OD values on infection efficiency. $\mathrm{h}$ Effect of seed germination status on infection efficiency ( 1 represents the seed that has not yet exposed the radicle; 2 represents the 
seed that has just sprouted the radicle; 3 represents that the seed has two cotyledons). i Effect of infection time on infection efficiency. Each treatment was treated with 20 seeds. The error bars represent the standard error $(n=3)$. Different letters indicate statistically significant differences at $P<0.05$ as determined by Duncan's test. Bars $=0.2 \mathrm{~cm}$.

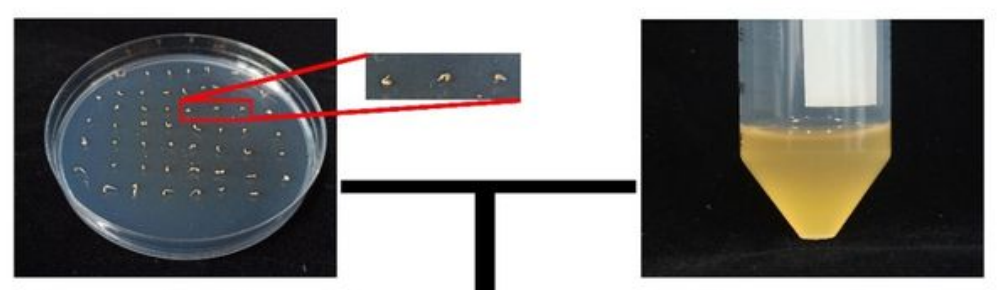

a. Seeds germination state (white small root tips)
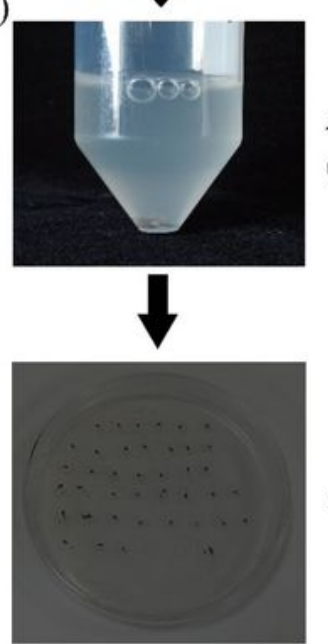

d. Dark culture for $3 \mathrm{~d}$

c. Submerge seeds in b. Prepare Agrobacterium Agrobacterium MS-infection $(\mathrm{OD}=1.5)$ for $24 \mathrm{~h}\left(28^{\circ} \mathrm{C}, 120 \mathrm{rpm}\right)$

DsRed

fluorescence

e. Screening by fluorescence microscope

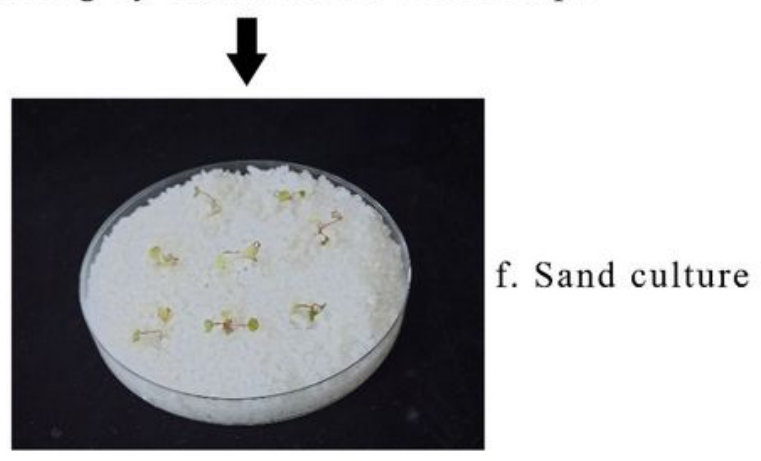

\section{Figure 3}

Transformation flow chart of FvCHLH-RNAi plants. 

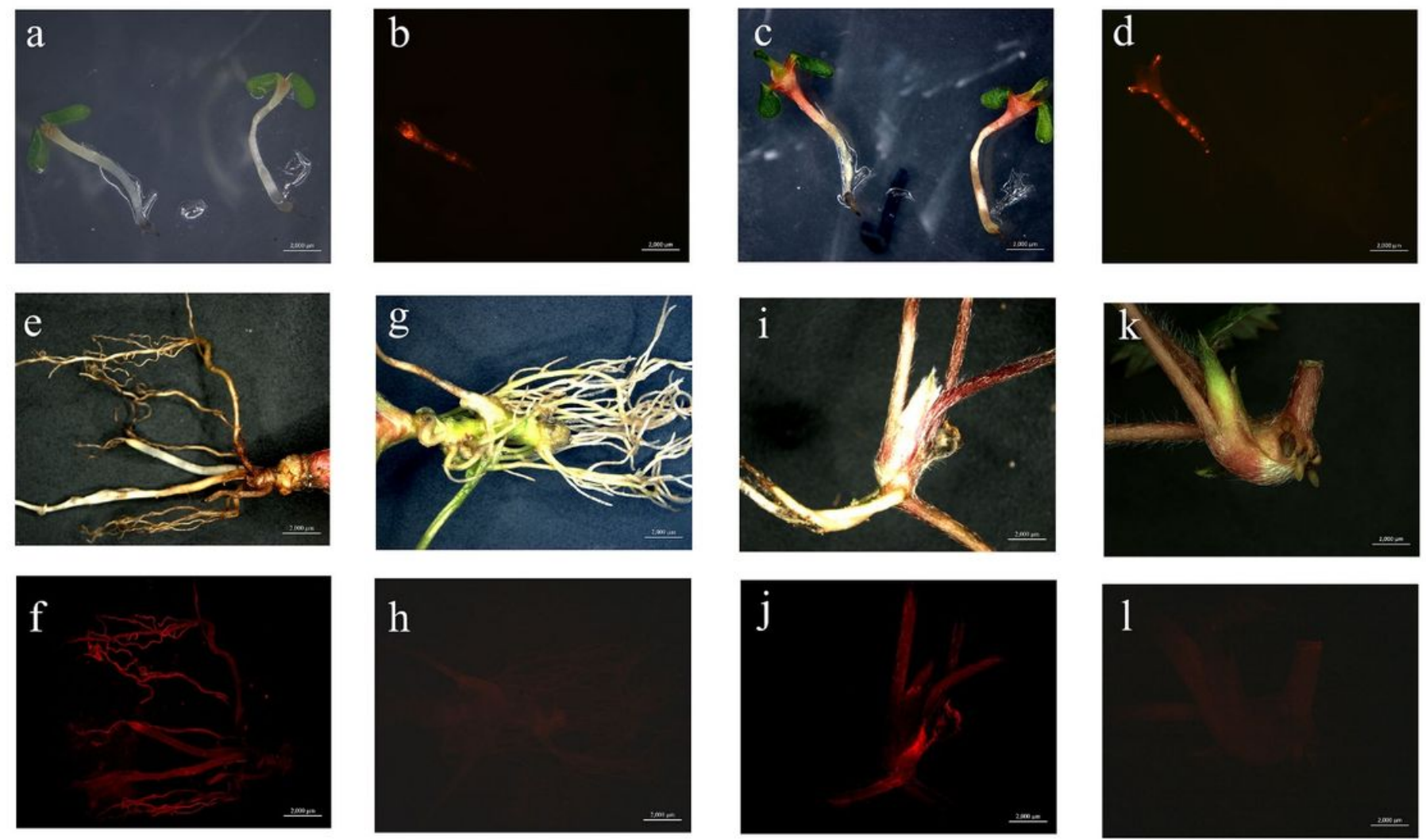

\section{Figure 4}

Expression of FvCHLH-RNAi plants red fluorescent protein. a and b 3-month-old transgenic plants (left) and 3-month-old wild-type plants (right). $\mathrm{c}$ and d 7-day-old transgenic plants (left) and 3-day-old wild-type plants (right). e and f 3-month-old transgenic plants roots. $g$ and $\mathrm{h}$ 3-month-old wild-type plants roots. $\mathrm{i}$ and $\mathrm{j}$ 3-month-old transgenic plants stolon. $\mathrm{k}$ and I 3-month-old wild-type plants stolon. 
a

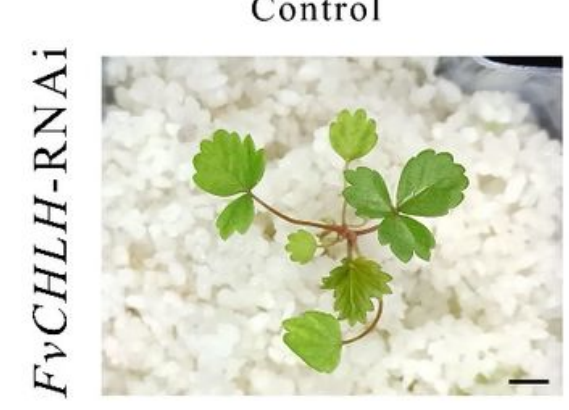

Phenotype-1

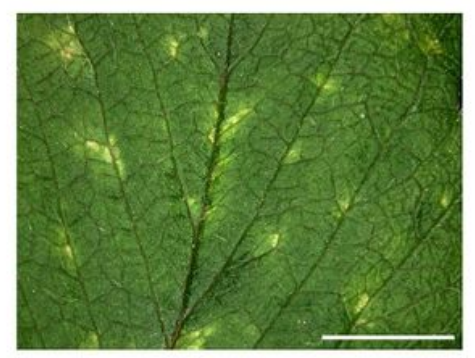

Phenotype-2

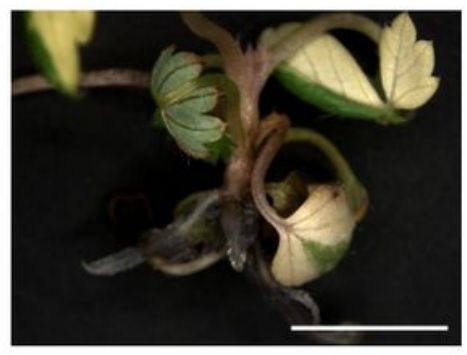

b

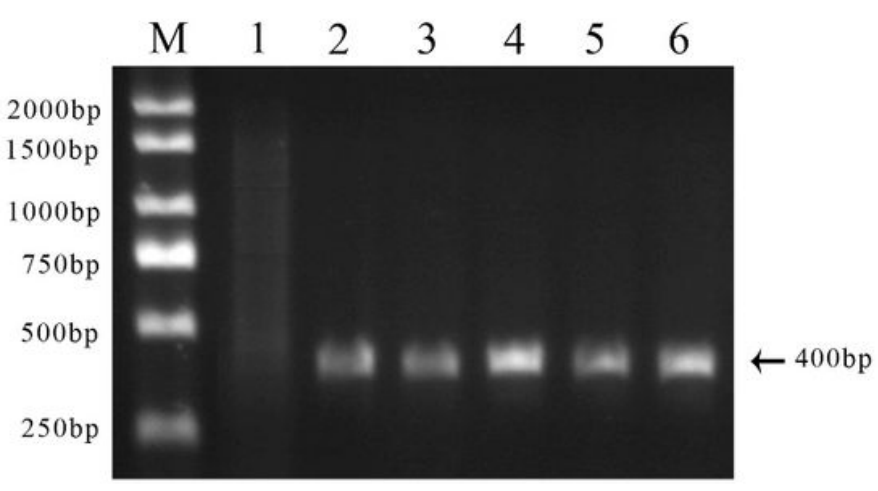

d

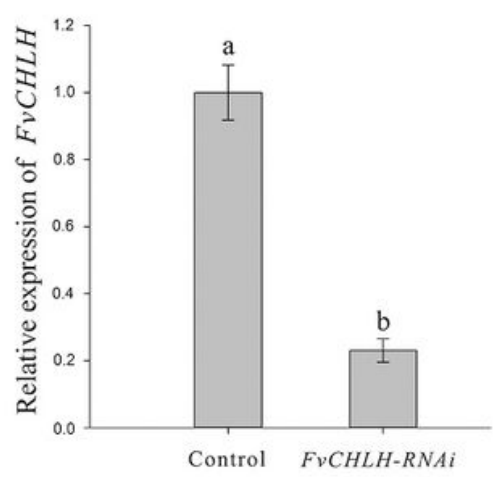

$\mathrm{C}$

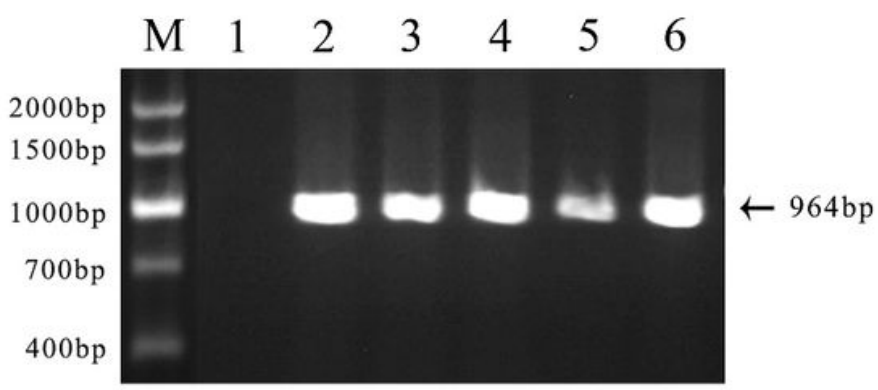

e

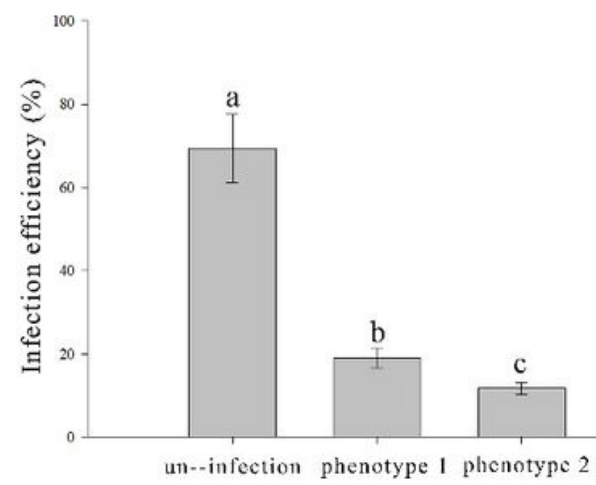

\section{Figure 5}

Phenotype of the FvCHLH-RNAi plants in strawberry. a Loss of green color in the FvCHLH-RNAi plants. b The 400-bp Kana products were detected in five FvCHLH -RNAi plants (line 2-6) but not detected in the wild-type plants (line 1) by qPCR. c The 964-bp DsRed products were detected in five FvCHLH -RNAi plants (line 2-6) but not detected in the wild-type plants (line 1) by qPCR. d FvCHLH gene expression of transgenic plants and wild type plants. e Proportion of un-infection plants, phenotype 1 and 2 plants to total infected plants. The error bars represent the standard error $(n=3)$. Different letters indicate statistically significant differences at $P<0.05$ as determined by Duncan's test. Bars $=0.5 \mathrm{~cm}$. 


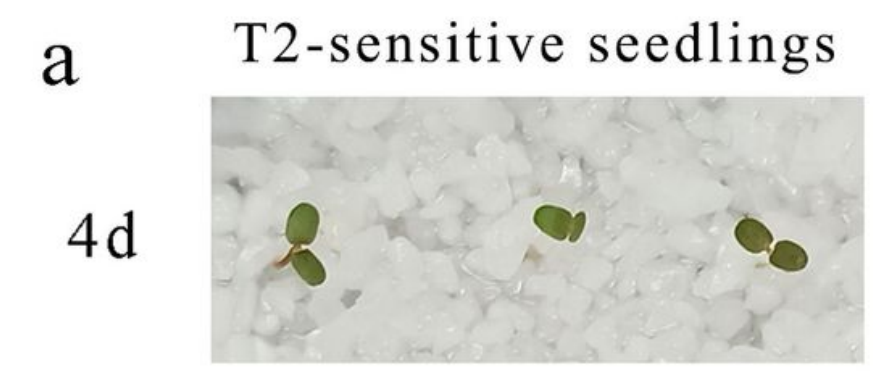

\section{T2-resistant seedlings}
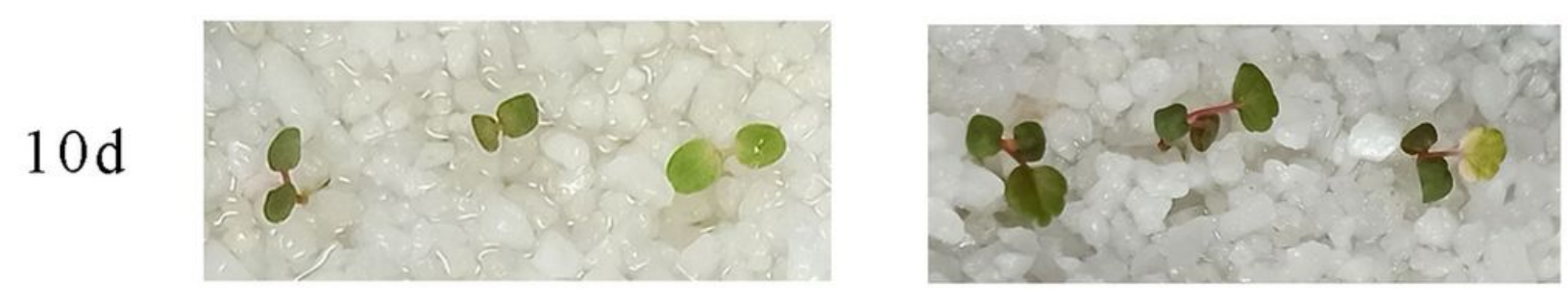

b
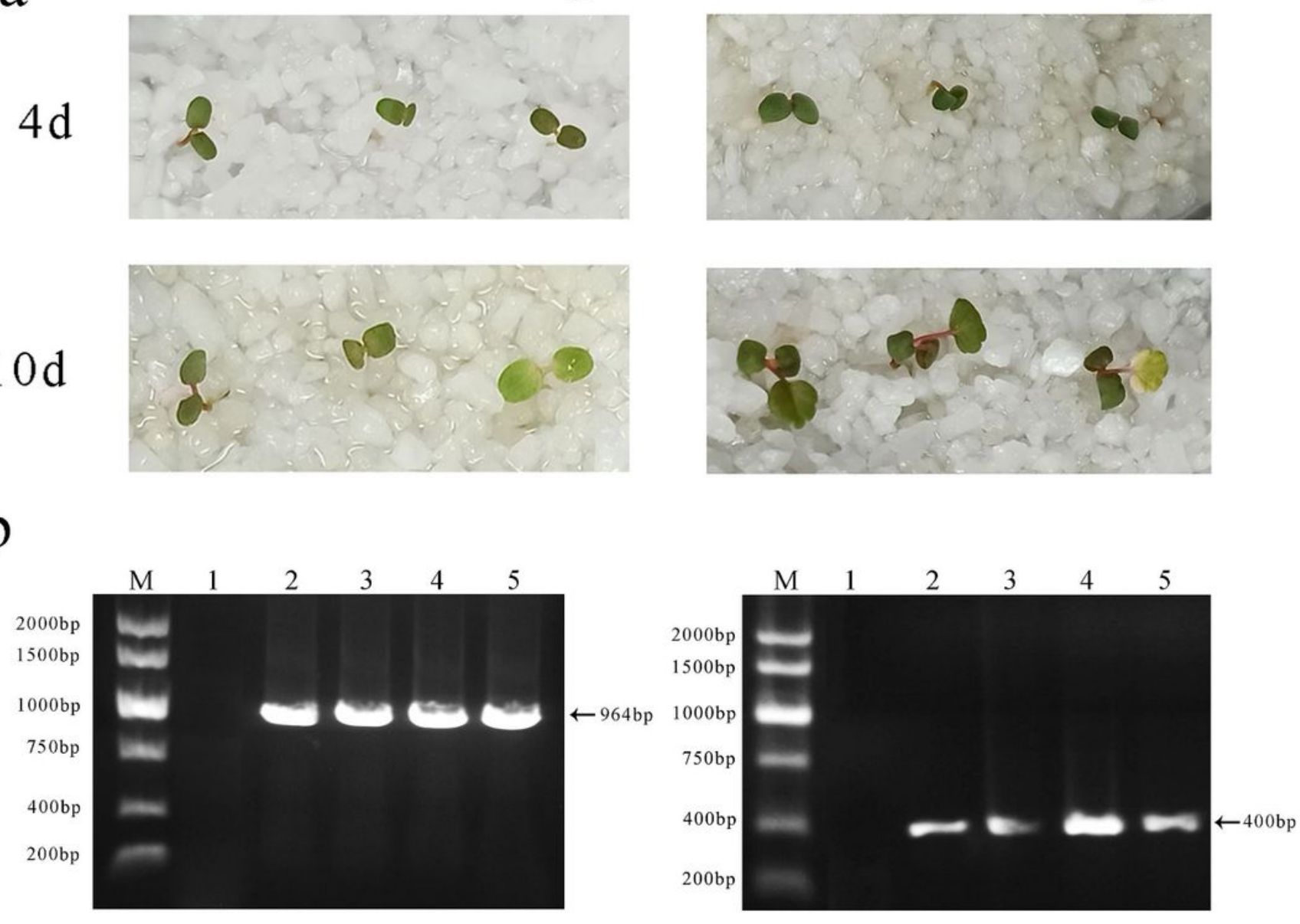

\section{Figure 6}

Isolation and identification of T2-resistant seedlings. a The phenotype of T2-sensitive seedlings for $4 \mathrm{~d}$ and $10 \mathrm{~d}$ under the irrigation of $75 \mathrm{mg} / \mathrm{L}$ kanamycin nutrient solution (left); the phenotype of T2-resistant seedlings for $4 \mathrm{~d}$ and $10 \mathrm{~d}$ under the irrigation of $75 \mathrm{mg} / \mathrm{L}$ kanamycin nutrient solution (right). $\mathrm{b}$ DsRed gene (left) and Kanamycin resistance gene (right) of T2-resistant seedlings of FvCHLH-RNAi plants. Line1 is T2-sensitive seedlings, line 2 is positive control, and line3-5 is T2-resistant seedlings. 